\title{
Development of Two Different Types of Correlative Light and Electron Microscope for Real Time Imaging and Quick Loading of Sample
}

\author{
In-Yong Park ${ }^{1, *}$, Moonseob Bae ${ }^{1}$ and Youngkwon Haam ${ }^{1,2}$ \\ 1. Division of Industrial Metrology, Korea Research Institute of Standards and Science (KRISS), 267 \\ Gajeong-Ro, Yuseong-Gu, Daejeon 34113, South Korea \\ 2. Department of Physics, Chungbuk National University, Seowon-Gu, Cheongju, Chungbuk 28644, \\ South Korea
}

Electron microscope (EM) can provide the images of structure with nanometer or sub-nanometer scale resolution easily, including properties of material by using an energy dispersive spectrometer. On the other hand, light microscope (LM) gives both images and colour information of species, but the resolution is generally limited to a few hundred nanometers. Recently, to combine the advantages of EM and LM, correlative light and electron microscopy (CLEM) is introduced [1-3]. Fluorescence microscope (FM) allows the users to recognize the particular point or area in the sample and study the cellular function in biology. Therefore, if the CLEM is comprised of EM and FM, it can analyse the molecules both in biological context and at nanometer imaging resolution. In this study, we present the two different types of CLEM. In light of LM, we integrate upright and inverted LMs with scanning electron microscopes (SEM) and show the experiment results.

To observe a same surface of sample with electron beam and light beam or an opaque sample, heretofore representative CLEM methods are rotating the sample with regard to each beam direction or drilling a small hole in the light objective lens for the electron beam to pass it through [4]. However, it is impossible to observe the sample simultaneously with two beams by first approach. In case of second approach, field of view of SEM would be reduced because of hole and it is important to minimize the charging while the electron passing through hole. In order to observe in real-time with two different beams, we place the reflecting mirror which has a hole for electron beam passing as figure 1 (b). A tungsten filament based SEM has a maximum magnification of about 50,000. The reflected wide-field fluorescence microscope provides $700 \mathrm{~nm}$ spatial resolution with $20 \times$ long working distance objective lens (Mitutoyo) which is positioned inside a vacuum chamber of SEM. Figure. 1 (c) shows the SEM image of metal grid and fig. 1 (d) shows the florescence image generated from quantum dots(QDs) attached on the metal grid.

For general SEM imaging, the sample should be placed in vacuum condition, so it take a time to pump out chamber for a sample preparation. Load-locks are applied in many modern SEM to reduce the time to transfer the sample from atmospheric to vacuum. However, it still needs a certain time including several chambers and valves. To shorten the sample loading and unloading time drastically, we designed the sample mount like an optical microscope slide glass. The shape of sample mount is same with slide glass as inset of fig. 2 (a), but the material is metal and has a small hollow hole, also the bottom side is covered a transparent glass. After placing a sample inside the hole, we put the sample mount into a small chamber which is positioned under an objective lens of SEM. The metal slide plate plays a role as not only a sample mount but also chamber door to maintain the vacuum condition. An inverted type optical microscope (Nikon ECLIPSE Ti-U) views the specimen through the glass window. In this way, SEM observe the upper side, while LM watch the lower side simultaneously as fig. 2 (c, d). After observation, sample unloading is just to pull out a sample mount from a small chamber. The sample in fig. 2 is also a 
metal grid coated with QDs. The maximum magnification of SEM is around 100,000 and LM supports the up to $100 \times$ objective lens.

In conclusion, we established two different types of CLEM. The first type allows the user to observe the sample in real-time with electron and light beams. The second type provides the quick sample loading and unloading based on inverted optical microscope. Proposed our apparatuses can provides a lot of information of species both high spatial resolution and fluorescence imaging easily and quickly. We have a plan to improve the CLEM system to observe the bio-sample based on low vacuum or combining with a cryo-sample preparation system, etc.

\section{References:}

[1] A. C. Zonnevylle, et al., J. Microscopy 252 (2013), p. 58-70

[2] C. J. Peddie, et al., Ultramicroscopy 143 (2014), p.3-14

[3] P. Boer et al., Nature methods 12 (2015), p. 503-513

[4] T. Kanemaru, et al., Ultramicroscopy 109 (2009), p. 344-349

The authors acknowledge funding from the financial support from the R \& D Convergence Program of NST (National Research Council of Science \& Technology) of Republic of Korea (Grant No.: CAP-143-KRISS).

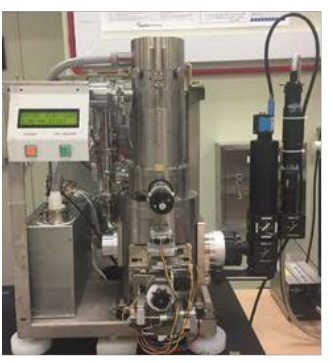

(a)

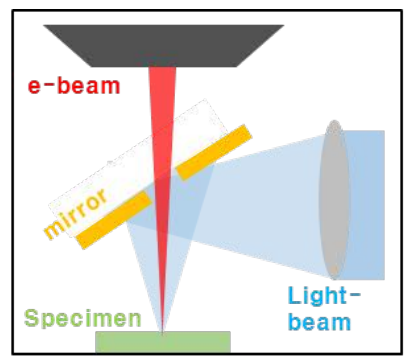

(b)



(c)



(d)

Figure 1. Upright type CLEM. (a) The picture of integrated system. (b) Schematic configuration. (c) The SEM image of metal grid. (d) The image of fluorescence image of metal grid covered with QDs



(a)

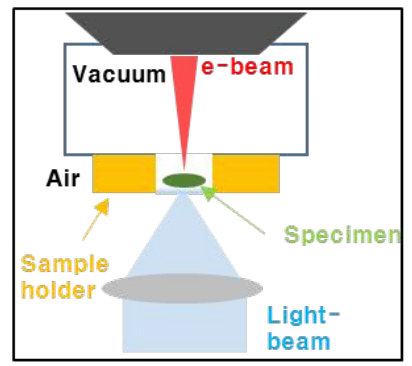

(b)



(c)

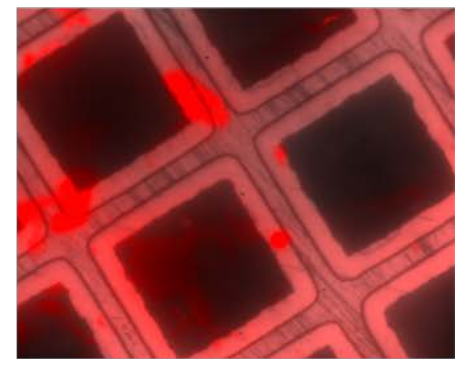

(d)

Figure 2. Inverted type CLEM. (a) The picture of integrated system. (b) Schematic configuration. (c) The SEM image of metal grid. (d) The image of fluorescence image of metal grid covered with QDs

\footnotetext{
*corresponding author: iypark@kriss.re.kr
} 